\title{
The Biofilm Community: Rebels with a Cause
}

\author{
A. Wilson Aruni • Yuetan Dou • Arunima Mishra • \\ Hansel M. Fletcher
}

Published online: 7 January 2015

(C) Springer International Publishing AG 2015

\begin{abstract}
Oral biofilms are some of the most complex and diverse ecosystems developed by successive colonization of more than 600 bacterial taxa. Development starts with the attachment of early colonizers such as Actinomyces species and oral streptococci on the acquired pellicle and tooth enamel. These bacteria not only adhere to the tooth's surface, but also interact with each other and lay foundation for attachment of bridging colonizers such as Fusobacterium nucleatum followed by late colonizers including the red complex species Porphyromonas gingivalis, Tannerella forsythia, and Treponema denticola, the founders of periodontal disease. As the biofilm progresses from supragingival sites to subgingival sites, the environment changes from aerobic to anaerobic, thus favoring the growth of mainly Gram-negative obligate anaerobes while restricting the growth of the early Gram-positive facultative aerobes. Microbes present at the supragingival level are mainly related to gingivitis and root caries, whereas subgingival species advance the destruction of teeth supporting tissues, and thus cause periodontitis. This review summarizes our present understanding and recent developments on the characteristic features of supra- and subgingival biofilms, interaction between different genera and species of bacteria constituting these biofilms, and draws our attention to the role of some of the recently discovered members of the oral community.
\end{abstract}

This article is part of the Topical Collection on Microbiology

A. W. Aruni $\cdot$ Y. Dou $\cdot$ A. Mishra $\cdot$ H. M. Fletcher $(\bowtie)$ Division of Microbiology and Molecular Genetics, School of Medicine, Loma Linda University, Loma Linda, CA 92354, USA e-mail: hfletcher@llu.edu

H. M. Fletcher

Institute of Oral Biology, Kyung Hee University, Seoul, Republic of Korea
Keywords Biofilms $\cdot$ Microbial $\cdot$ Periodontal disease . Non-bacterial microbiota

\section{Introduction}

Microbial colonization begins immediately following birth and initiates a symbiotic relationship between the microorganisms and the host $[1,2]$. Dysbiosis of these communities are linked to various disease states [3, 4]. While it is estimated that the majority of the total microbial biomass lives in the planktonic mode [5••], more than $65 \%$ of all bacterial infections in humans are biofilm associated $[6,7]$. Oral bacterial biofilms were the first human-associated biofilms to have been studied extensively. First defined by James Leon Williams in 1897, dental biofilm was described as a gelatinous accumulation of bacteria attached to the tooth surface [8]. Recent statistics show that oral diseases affect 3.9 billion people globally [9]. Periodontitis, as one example, is a biofilm-associated infection that induces an irreversible inflammatory state that leads to the destruction of the supporting structures of the teeth [10]. The interactions among the biofilm communities are defined spatiotemporally. Since biofilms exist as an interface, their environment is characterized by a gradient of nutrients that could encourage spatial and metabolic diversity promoting growth of the microbiota. Thus, in the current emerging paradigm, periodontitis is considered a multifactorial disease with diverse clinical features not precisely explained by the etiologic role of a single bacterium.

This microbial accumulation that adheres tenaciously to the tooth's surface shows structural organization, partly due to the organic matrix-derived salivary glycoproteins and extracellular microbial products [11]. This accumulation is broadly 
classified as supragingival and subgingival biofilm. Supragingival biofilm, sometimes referred to as "marginal biofilm", is found at or above the gingival margin. In contrast, subgingival biofilm is found below the gingival margin, between the tooth and gingival sulcular tissue. The different regions of biofilm are involved in significant processes associated with different diseases of the teeth and periodontium. Therefore, addressing gingival biofilms is important in the prevention of oral infections. Marginal biofilm, which predisposes to gingivitis, and tooth-associated subgingival biofilm are critical in calculus formation and root caries, whereas subgingival biofilm is involved in soft tissue destruction and could lead to different forms of periodontitis. Although not understood as biofilms, descriptions of supra- and subgingival dental biofilms were available in the 1960s to 1970s [5••]. Since that era, there have been noticeable advances in knowledge on the biofilm mode of growth causing periodontal infections. The composition of these biofilms predominate with bacterial microbiota; however, nonbacterial microorganisms such as mycoplasma, yeast, protozoa, viruses, and fungi are a part of this community [2]. Furthermore, they also contain host cells such as epithelial cells, macrophages, and leukocytes. The major extracellular matrix which supports the biofilm mass consists of organic and inorganic materials derived from saliva, gingival crevicular fluid, and bacterial products. The organic components of the matrix include glycoproteins (from saliva), polysaccharides (produced by bacteria), albumin (from crevicular fluid), and lipid materials (from disrupted bacterial and host cells) [12]. Biofilm formation can be divided into three phases, starting with initial formation of the pellicle coating on the tooth surface, followed by initial colonization of bacteria, and then secondary colonization and biofilm maturation [13].

Earlier studies on the stages of dental biofilm development and maturation on the tooth surface have shown that the communities grow by lateral spread and gain thickness due to the multiplication of the early colonizers [11]. Competition for space and nutrition facilitate aggregation of different bacterial species. In mature biofilms, a columnar pattern of microbial growth have demonstrated that certain bacterial species under specific environmental conditions become organized into supragingival and subgingival biofilms with unique characteristics. Hence, this biofilm mode growth implies that such bacterial colonies behave as integrated communities and play a role in chronic biofilm-associated infections. Thus, the emerging paradigm supports the induction of disease due to pathogen synergy [14].

Cultivation techniques and molecular approaches have shown the width and depth of biofilm diversity, and a vast collection of microorganisms that inhabit the gingival crevices [15]. These investigations give a detailed taxonomical census of gingival microbial communities, including subject variation and temporal variation in community structure, determining periodontal health status. However, understanding its community function is yet to be explored in detail, and clinical studies and evaluation of the ecological determinants of gingival biofilm maturation are still needed to address this issue. Functional characteristics of gingival biofilm communities are governed by the metabolic processes that support such microbial communities [16]. Thus, identifying keystone species, along with their microbial interactions and signaling, play a significant role in understanding the relationship between microbial communities and the host $[17,18]$.

\section{Early and Late Colonizers}

Gingival biofilm formation is a progressive phenomenon with sequential addition of specific groups of bacteria to the glycoprotein complex on the surface of the teeth. The initial predominant bacteria that colonize the pellicle coated tooth surface are Gram-positive facultative anaerobic bacteria such as Actinomyces spp and oral streptococci [12]. Streptococci are considered to be the main group of early colonizers in the oral biofilm, making up over $80 \%$ of the initial biofilm. They interact with other primary colonizers and attach to the tooth surface, determining the composition of late colonizers in the oral biofilm, and impacting the health or disease status of the host $[17,18]$. These initial colonizers adhere to the pellicle through specific molecules termed adhesins on the bacterial surface that, in turn, interact with receptors on the dental pellicle. As a continuous process, there is a transition from the early aerobic environment characterized by Gram-positive facultative anaerobic species to a highly oxygen deprived environment where Gram-negative anaerobic microorganisms dominate $[19 \bullet, 20]$.

Further to the inhabitation of early colonizers, secondary colonizers such as Prevotella intermedia, P. loescheii, Capnocytophaga spp, and Fusobacterium nucleatum adhere to bacteria already present in the biofilm matrix, and subsequently attract late colonizers such as Porphyromonas gingivalis [21, 22••]. Extensive laboratory studies have documented the ability of different species and genera of biofilm microorganisms to adhere to one another, a process known as "coaggregation" [23]. This process occurs primarily through the highly specific stereochemical interaction of protein and carbohydrate molecules located on the bacterial cell surfaces, in addition to the less specific interactions resulting from hydrophobic, electrostatic, and van der Waals forces [24]. Supragingival biofilm typically demonstrates a stratified organization of the bacterial morphotypes. Gram-positive cocci and short rods predominate at the tooth surface, whereas Gramnegative rods and filaments as well as spirochetes do so in the outer surface of the mature biofilm mass [25]. Highly specific cell-to-cell interactions forming the "corncob" structures are also evident. Corncob formations have been observed between 
rod-shaped bacterial cells (e.g., Bacterionema matruchotii or F. nucleatum) which form the inner core of the structure and coccal cells (e.g., Streptococci or P. gingivalis) that attach along the surface of the rod shaped cell [16].

Members of the Mitis group Streptococci (Streptococcus oralis, S. mitis, and S. sanguinis) are some of the early colonizers and have been frequently associated with oral health $[26,27]$. It is the uncontrolled growth of the Gram-negative component of dental biofilm that leads to periodontitis. During progression of periodontitis, there is a reduction in the fraction of streptococci present in the biofilm. Studies have shown that $P$. gingivalis induces $S$. mitis cell death (10-fold increase) and DNA fragmentation, and increases production of reactive oxygen species by up to 25 -fold in an in vitro biofilm system by an unknown mechanism, thus shaping the oral microbiome to its advantage [28]. Therefore, it could be reasoned that successive biofilm formation may play a role in major periodontal species pathogenicity.

During the initial oral biofilm formation process, oral Veillonella species (Veillonella atypica, V. denticariosi, $V$. dispar, V. parvula, V. rogosae, and V. tobetsuensis) are the major early colonizers [29]. The interaction of Veillonella with certain other bacterial species via several factors is important for enhanced biofilm development. Biofilm mass was shown to be increased when selected Veillonella species were co-cultured with $S$. gordonii, S. mutans, or $S$. salivarius compared to monoculture controls [29]. In contrast, the reduced biofilm mass shown by co-culture of S. sanguinis, with selected Veillonella species may indicate the role of specific factor(s) including signaling molecules in the heterogeneous response [29]. Further studies are needed to clarify the molecular mechanism(s).

\section{Biofilm and Periodontal Disease}

Despite the significance of biofilm in periodontal infections, there have been varied thoughts on the composition of subgingival microbiota resulting in tissue destruction, and hence, not all the elements of biofilm may have the same significance in causing the disease process. Supragingival and subgingival biofilms are always considered a continuum; however, at a certain point during the biofilm maturation process, the subgingival habitat is isolated from the supragingival environment. The lack of complete interdependence between these two ecosystems is consistent with the observation that removal of the supragingival biofilm has a minimal impact on the composition of the subgingival biofilm and its capacity to play a role in periodontal disease [30]. This observation could suggest that after a certain point of biofilm development and maturation, the subgingival microbiota does not depend on the supragingival bacterial species for its survival and pathogenicity. Earlier studies have also shown that there was a vast variation in the healthy biofilm community versus the diseased and that certain components in this community can play a relevant role in the disease process.

Periodontal disease induced by the dysbiosis can be summarized by the "ecological biofilm hypothesis". Based on this hypothesis, changes in the oral environment increase the competitiveness of the pathogen at the expense of the species associated with oral health, and hence, increases the expression of virulence factors in that organism [20,19•]. This also takes into consideration the interplay between bacteria and the host inflammatory response. Therefore, maturation of supraand subgingival biofilms leading to gingival inflammation would result in additional changes in the microbial composition of the adjacent biofilm that could also be regulated by reciprocal interaction of the host [31]. While the factors in the subgingival environment can affect the biofilm composition, environmental conditions such as temperature, redox potential, and $\mathrm{pH}$ availability on nutrients, can also alter pattern of gene expression of certain pathogenic bacterial strains in the biofilm $[32,33]$. Therefore, the local microenvironment plays a vital role in the composition and function of the subgingival microbiota [34]. A shift in the microbial community during experimental gingivitis is correlated with changes in the microbial population in a developing dental biofilm $[35,36]$. During periodontal infection, an increase in gingival crevicular fluid causes early changes in the bacterial composition of supragingival biofilm that become more noticeable as the flora matures. This, in turn, is further perpetuated by autogenic microbial succession and regulated microbiota interactions [31]. While dysbiosis has also been used to describe disease state due to a shift in the normal resident microbiota, previous studies have examined the changes in the microbiota from different oral habitats over a time period [37, 38••, 39-41, 42•, 43]. These studies have taken into account other environmental factors in microbial succession and facilitated the understanding of the microbial interplay.

Based on the evidence and significance of gingival biofilms, the primary goal of periodontal therapy is to target the subgingival biofilm formation at the diseased sites. Understanding these bacterial biofilm communities will provide knowledge to design new treatments for chronic infectious diseases. It was also noted that supragingival biofilm regulation plays a critical role in therapy due to its impact on the inhibition of re-colonization in the subgingival biofilm [44-46]. It was also noted that there have been conflicting results regarding the impact on the control of supragingival biofilm on the composition of subgingival biofilm in untreated periodontal sites [47-49].

\section{Supragingival Biofilms}

Earlier studies have shown the succession of bacterial species in the supragingival biofilm samples during different time 
frames starting within hours [40] to days [42•, 43] and months [37] after professional cleaning. These studies have confirmed the adhesion and specific attachment of early colonizers to the tooth surface by bacteria classified as the "yellow complex" $S$. intermedius, S. oralis, and S. mitis [40]. After biofilm removal, re-colonization of the supragingival environment with species such as V. parvula, C. gingivalis, E. corrodens, Neisseria mucosa, and F. nucleatum has been noted [43]. There was an increase in the bacterial counts in supragingival biofilms in periodontitis subjects compared to healthy ones, though both the clinical groups presented a similar pattern of microbiota [43]. Treponema sokranskii, S. noxia, S. mutans, $S$. anginosus, P. melaginonenica, Propionibacterium acnes, $N$. mucosa, and Leptotrichia buccalis were the predominant bacteria noted in the healthy as well as the periodontitis supragingival biofilm samples $[30,11]$. A study using cluster analysis and community orientation techniques to evaluate microbial complexes among grown biofilm showed a mixture of biofilm communities that appeared similar to the more mature and long-term biofilms [37]. However, the studies could not identify the community of late colonizers. Communities including Streptococcus (yellow complex) and Actinomyces species similar to those described for subgingival biofilms could be identified [50]. However, for other complexes such as "green", "purple", "orange", and "red complexes", there were differences between the supra- and subgingival biofilms [51]. Capnocytophaga ochracea appeared to be associated with more members of orange complex bacteria. V. parvula, and $N$. mucosa formed a new "purple complex" where $N$. mucosa replaced A. odontolyticus. The largest complex observed in the supragingival samples in terms of number of species was the "orange complex". There were distinct subsets within the "orange complex", such as C. gracilis, S. noxia, C. ochracea, P. intermedia, P. nigrecens, and certain Fusobacteria formed additional subsets such as C. retus and C. showae [37]. It is likely that this could be due to similar nutritional requirements of the bacterial subset community. It was also noted that the subsets within the "orange complex" of supragingival biofilms seemed to be more closely associated with those observed in the subgingival samples. Regarding the "red complex" bacteria species, $P$. gingivalis, Tannerella forsythia, and $T$. denticola were noted in association with $E$. nodatumin supragingival biofilms in both mature and long-term biofilm complexes. Further, there was an association of "red complex" with Aggregatibacter actinomycetemcomitans, E. saburreum, Parvimonas micra, and P. melaninogenica in the long-term biofilm complexes. Studies on the impact of biofilm biomass and tooth position on supragingival biofilm composition indicated that "green" and "orange" complex bacteria increased markedly in samples with high biofilm mass [11]. Additionally, it is important to note that the members of Actinomyces and "purple complexes" decreased, but the "red complex" species in the supragingival biofilms was not impacted by the biofilm biomass. Gingival crevicular fluid and surface type were found to be major limiting factors for biofilm development and redevelopment [52•]. Biofilm redevelopment was more rapid in the natural teeth as compared to dentures [42•]. Also, the roughness of the colonization surface was important for biofilm colonization other than the physiochemical properties $[53,54]$. Recent studies have shown that A. actinomycetemcomitans, A. baumanni, and the "red complex" (P. gingivalis, T. forsythia, T. denticola) associated with $P$. aeruginosa in the subgingival microbiota increased the likelihood of periodontitis [55]. Also, P. aeruginosa showed synergism with $A$. actinomycetemcomitans in increasing the risk of periodontal disease.

Recent studies have indicated that supragingival biofilm can harbor putative periodontal pathogens suggesting a possible role of this environment as a reservoir for spread or reinfection to subgingival sites. Forty taxa of bacteria were detected in both the supra- and subgingival biofilms with Actinomyces spp. being the most prevalent one in both habitats [56]. Supragingival samples exhibited significantly higher counts of $A$. naeslundii genospecies 1, A. israelii, A. odontolyticus, N. mucosa, S. gordonii, C. ochracea, and C. sputigena compared to the subgingival samples taken from the same tooth surface [57]. Subgingival biofilm samples showed higher counts of $P$. nigrescens, $P$. intermedia, Bacteroides forsythus, and $P$. gingivalis. They exhibited a higher proportion of "red" and "orange" complex species while supragingival biofilm exhibited a higher proportion of "green" and "purple" complex species as well as Actinomyces spp. [56]. In summary, supragingival colonization was initiated by "yellow" complex bacteria such as $S$. mitis and $S$. oralis and the colonization of Actinomyces spp. was slower. The development and composition of the supragingival microbiota is influenced by inflammation and depth of periodontal pockets [11]. Other factors include the nature of surfaces, tooth position, and biofilm mass [38••].

\section{Subgingival Biofilms}

The environmental parameters of the subgingival region differ from those of the supragingival region. The gingival crevice or pocket is bathed by the flow of crevicular fluid, which contains many substances that the bacteria may use as nutrients. Host inflammatory cells and mediators are likely to have considerable influence on the establishment and growth of bacteria in this region. The bacteria that predominate in mature biofilms are anaerobic, asaccharolytic, and use amino acids and small peptides as energy sources [58]. Laboratory studies have demonstrated many physiologic interactions among bacteria found in dental biofilm [25]. The host also functions as an important source of nutrients. For example, hemin iron from 
the breakdown of host hemoglobin may be important in the metabolism of $P$. gingivalis [59]. This is one of the important host components that govern the relative proportion of subgingival biofilm. Increases in steroid hormones are associated with significant increases in the proportions of P. intermedia found in subgingival biofilm [60].

Among the subgingival consortia, Gram-negative anaerobes such as $P$. gingivalis, T. forsythia, and T. denticola, are characteristic bacteria associated with periodontal disease rather than health. These are also clustered as the "red complex" species [28]. Studies investigating colonization of human gingival multi-layered epithelium by 10 -species subgingival biofilm (F. nucleatum, Campylobacter rectus, V. dispar, P. gingivalis, P. intermedia, T. forsythia, T. denticola, A. oris, S. anginosus, and $S$. oralis) or its 7 -species variant lacking $P$. gingivalis, T. forsythia, and T. denticola, evaluated the relative effects of the "red complex" species on the composition and cellcolonizing capacities of the remaining seven species [61]. Results showed that though the "red complex" species colonized well in gingival epithelia, their absence from the biofilm increased the colonization of $S$. oralis. This antagonistic interaction with streptococci shows that the "red complex" may have an inhibitory role on the colonization ability of other bacterial species in the biofilm, and thus may regulate the virulence properties of the whole biofilm community [61].

Recent studies showed that Fusobacterium and Treponema were among the most abundant subgingival genera and the phyla Spirochetes and Synergistetes were increased in periodontitis [62, 58]. Another study showed increased abundance of Bacteroidetes along with Fusobacterium and Treponema in periodontitis, and high proportions of periodontitis-associated Prevotella was also observed $[62,58]$. Additionally, it was interesting to note that some Streptococcus OTUs such as $S$. sanguinis were increased in health and that sequences related to $S$. mitis remained unchanged, whereas $S$. constellatus and an uncultured Streptococcus spp. (OT 071) were associated with disease. Physiological differences that could explain such heterogeneity in niches occupied by streptococci are not currently known. Although this study showed that Actinobacteria was strongly associated with health [62], an earlier study indicated that Proteobacteria was the only healthassociated phylum [58]. Taken together, specific microbial combinations in the subgingival biofilm community are associated with health or disease.

\section{Pathogen Synergy and Bacterial Community Dynamics}

Early colonizers such as Streptococcus and Actinomyces spp. use oxygen and lower the redox potential of the environment that favors anaerobic species [12]. Gram-positive early colonizers use sugars as an energy source. The bacteria that predominate in mature biofilm are anaerobic and asaccharolytic and use amino acids and peptides as energy source [63]. There has been a strong metabolic interaction among the different bacteria found in the dental biofilm. Lactates and formates are the byproducts of metabolism of Streptococci and Actinomyces spp. These molecules are also used by other organisms like Veillonella spp. and $A$. actinomycetemcomintans $[64,65]$. The growth of $P$. gingivalis is enhanced by succinate-producing $C$. ochracea and protoheme-producing $C$. rectus [66-68]. Thus, the biofilm community seems more efficient at releasing energy from the different bacterial-derived available substrates based on their specific requirements and environmental changes. In addition, host-derived substrates present in saliva and crevicular fluid are also important energy sources. For example, certain bacterial enzymes from the arginine degradation cycle can generate an important nitrogen source mediated by the release of ammonia from the degradation of protein components in saliva and crevicular fluid. Bacteria such as Filifactor alocis have a well-developed arginine metabolic pathway that is noted to be upregulated during co-infection with P. gingivalis $[69,70 \bullet]$.

Since most biofilm-forming bacteria are primarily asaccharolytic, but resort to protein breakdown for energy and survival, this process could lead to a heavy production of ammonia. A well-developed arginine cycle noted in many bacteria such as F. alocis could help in pathogen synergy and survival in periodontal pockets by using the toxic metabolites of the host cell. The F. alocis arginine metabolic pathway predicts the enzymatic degradation of arginine by arginine deaminase, leading to the conversion of arginine to ornithine and ammonia [71]. Arginine degradation could favor increase in the $\mathrm{pH}$ that would counteract acidic conditions generated from carbohydrate catabolism in a mixed bacterial oral flora. In the periodontal pocket, these amino acids can also be available from the degradation of various protein substrates by the biofilm community and host-derived proteases for their nutritional support, survival and virulence [72].

It is evident that community synergism between organisms in the biofilm compensate the energy source for survival and copathogenesis. Also, hemin iron from the breakdown of host hemoglobin may be an important process utilized by $P$. gingivalis [73]. Additionally, studies have shown that an increase in the steroid hormones is associated with increase in organisms like P. intermedia found on the subgingival biofilm [74]. Therefore, nutritional interdependences seem to play a critical role in growth and survival of dental biofilm bacteria which could explain the structural interactions observed among them.

\section{New Candidates and Yet Culturable Bacteria}

The human microbiome has been established with the goal to characterize the bacterial communities associated with 
different body sites [75]. The Human Oral Microbiome Database (HOMD) [76] and the CORE database [77•] contain information on the prokaryotic species present in the oral cavity. The HOMD contains approximately 619 validated taxa with 1,178 total taxa identified, of which $24 \%$ are named, $8 \%$ are cultivated, but unnamed, and $68 \%$ are uncultivated phylotypes [78]. Collectively, these, as well as other oral microbiome studies conducted over the last several years, have modified and enhanced our understanding of the oral microbial communities in health and disease. Current theories on the etiology of periodontitis favor a shift in microbial composition that is caused by a decrease in beneficial symbionts and an increase in organisms with enhanced pathogenic potential. Thus, in the biogenesis of the disease there is an increase in microbial diversity, its composition and pathogenic communities. Moreover, these communities contain higher levels of fastidious and yet-to-be-cultivated taxons than previously recognized $[78,58]$.

The important "red complex" bacteria along with other cultivable bacteria species such as $P$. intermedia, A. actinomycetemcomitans, F. nucleatum, Selenomonas noxia, and Eubacterium nodatum are associated with periodontitis $[21,22 \bullet \bullet, 79]$. In addition, organisms such as Selenomonas, Synergistes, Desulfobulbus, TM7 (new candidate bacterial division), and $F$. alocis have been identified as potential pathogens in a number of independent studies [78, 58]. Moreover, $20-60 \%$ of the phylotypes identified in the oral cavity are yet to be cultivated $[78,58]$. This has raised questions on the relative significance of these microbes in the disease process. New organisms of the biofilm community have been shown to participate in their unique characteristics, virulence potential and capacity to act in community dynamics, which were proven crucial in periodontitis.

Traditional culture-based studies and general molecular surveys, however, offered limited information on the total diversity present in the subgingival environment. A recent molecular survey performed using pyrosequencing of $16 \mathrm{~S}$ rRNA gene provided a broader picture identifying overall difference in relative abundance of more than 700 subgingival species-level taxa, confirming the association of $P$. gingivalis, T. denticola, and T. forsythia with periodontitis, and revealing new species. Among them, F. alocis is strongly associated with disease [58]. Studies have shown that candidate division TM7 bacteria are detected in biofilm metagenome and this bacterium, when grown in dual species biofilm showed pleiomorphic phenotypes. The TM7 bacteria formed long filaments with $A$. oris or $F$. nucleatum, but grew as short rod or cocci with $P$. gingivalis, $P$. intermedia, $P$. micra, or $S$. gordonii [80]. Also, the TM7 bacteria were shown to be closely associated with A. oris, F. nucleatum, P. gingivalis, P. intermedia, P. micra, and S. gordonii [80]. The importance of $F$. alocis is noted as it is present in high abundance in the periodontal pocket, shows community synergism, and is involved in oral biofilm formation with other important periodontopathic bacteria $[81,70 \bullet, 82]$. Therefore, the composition of these biofilms may determine the severity of the disease as their interactions are important for host modulation.

\section{Non-Bacterial Microbiota}

In the current emerging paradigm, periodontitis is considered a multilayered multifactorial disease with diverse clinical features not precisely explained by the etiologic role of bacteria. In addition to bacteria, viruses occupy a unique position and have been associated with destructive periodontal diseases [83-89]. Since the number of viruses in the oral cavity is high, their role in periodontitis and host modulation is under study. These two important microbial entities, namely viruses and bacteria, have developed diverse mechanisms to affect directly host cell epigenetics driving pathogenesis and oncogenesis [90-93]. It is noteworthy that certain bacteria and viruses show mutual relationship affecting the host. Certain viruses bind to bacterial polysaccharides and adhesion proteins to establish productive infection [94]. Studies have shown that bacterial adhesion molecules such as MSCRAMMs could play a triple role in virus-bacteria-host interactions [94]. Viruses themselves can form extracellular assemblies that resemble bacterial biofilms in composition, organization, and dissemination $[84,85]$. Our earlier studies using a co-infection model with major biofilm-forming periodontopathic bacteria $F$. alocis and $P$. gingivalis have shown expression of MSCRAMMs. Such MSCRAMMs could act as potential ligands for viruses to interact, resulting in a wider network of biofilm microbiota [94].

\section{Summary and Conclusions}

In recent years, many new members of oral biofilm have been identified such as Selenomonas, Synergistes, Desulfobulbus, TM7 (new candidate bacterial division), and $F$. alocis. The molecular basis of the interaction of these bacteria with already established ones is not clearly understood. Studies have just begun to understand the initial interactions of these organisms with other community members. Much effort is needed to determine their behavior, virulence potential, and to give these individuals a specific place in a complex community. In the oral cavity, $20-60 \%$ of the phylotypes are yet to be cultivated. Thus, the major future challenge of the field is to develop new research approaches to fully characterize these bacteria. Since they represent a significant percentage of the oral microbial population, the gap in our comprehensive understanding of their pathogenesis and specific role in the biofilm community will compromise the development of new effective measures to control periodontitis and other 
biofilm-associated diseases. Future molecular studies in this area are of critical importance.

Acknowledgments The authors' work cited in the manuscript was supported by Public Health Services Grants DE13664, DE019730, DE019730 04S1, DE022508, DE022724 from NIDCR (to H.M.F.).

\section{Compliance with Ethics Guidelines}

Conflict of Interest A. Wilson Aruni, Yuetan Dou, Arunima Mishra, and Hansel M. Fletcher declare that they have no conflict of interest.

Human and Animal Rights and Informed Consent This article does not contain any studies with human or animal subjects performed by any of the authors.

\section{References}

Papers of particular interest, published recently, have been highlighted as:

- Of importance

- Of major importance

1. Dethlefsen L, McFall-Ngai M, Relman DA. An ecological and evolutionary perspective on human-microbe mutualism and disease. Nature. 2007;449:811-8.

2. Avila M, Ojcius DM, Yilmaz O. The oral microbiota: living with a permanent guest. DNA Cell Biol. 2009;28:405-11.

3. Said HS, Suda W, Nakagome S, et al. Dysbiosis of salivary microbiota in inflammatory bowel disease and its association with oral immunological biomarkers. DNA Res. 2014;21:15-25.

4. Jones ML, Martoni CJ, Ganopolsky JG, et al. The human microbiome and bile acid metabolism: dysbiosis, dysmetabolism, disease and intervention. Expert Opin Biol Ther. 2014;14:467-82.

5.•• Bjarnsholt T. Introduction to biofilms. New York: Springer; 2011.

6. Lewis K. Persister cells and the riddle of biofilm survival. Biochemistry (Mosc). 2005;70:267-74.

7. Lewis K. Riddle of biofilm resistance. Antimicrob Agents Chemother. 2001;45:999-1007.

8. Cassai E, Galvan M, Trombelli L, et al. HHV-6, HHV-7, HHV-8 in gingival biopsies from chronic adult periodontitis patients. A casecontrol study. J Clin Periodontol. 2003;30:184-91.

9. Kassebaum NJ, Bernabe E, Dahiya M, et al. Global Burden of Severe Periodontitis in 1990-2010: A Systematic Review and Meta-regression. J Dent Res. 2014;93:1045-53.

10. Bergstrom J. Periodontitis and smoking: an evidence-based appraisal. J Evid Based Dent Pract. 2006;6:33-41.

11. Teles R, Teles F, Frias-Lopez J, et al. Lessons learned and unlearned in periodontal microbiology. Periodontol 2000. 2013;62:95-162.

12. Marcotte H, Lavoie MC. Oral microbial ecology and the role of salivary immunoglobulin A. Microbiol Mol Biol Rev. 1998;62:71109.

13. Brogden KA and Guthmiller JM. Polymicrobial diseases. 2002

14. van Steenbergen TJ, van Winkelhoff AJ, De GJ. Pathogenic synergy: mixed infections in the oral cavity. Antonie Van Leeuwenhoek. 1984;50:789-98.

15. Diaz PI. Microbial diversity and interactions in subgingival biofilm communities. Front Oral Biol. 2012;15:17-40.

16. Zijnge V, Ammann T, Thurnheer T, et al. Subgingival biofilm structure. Front Oral Biol. 2012;15:1-16.
17. Hajishengallis G, Darveau RP, Curtis MA. The keystone-pathogen hypothesis. Nat Rev Microbiol. 2012;10:717-25.

18. Kolenbrander PE. Oral microbial communities: biofilms, interactions, and genetic systems. Annu Rev Microbiol. 2000;54:413-37.

19. Marsh PD. Microbial ecology of dental plaque and its significance in health and disease. Adv Dent Res. 1994;8:263-71.

20. Marsh PD. Are dental diseases examples of ecological catastrophes? Microbiology. 2003;149:279-94.

21. Kolenbrander PE, Andersen RN, Blehert DS, et al. Communication among oral bacteria. Microbiol Mol Biol Rev. 2002;66:486-505.

22.• Kolenbrander PE, Palmer Jr RJ, Rickard AH, et al. Bacterial interactions and successions during plaque development. Periodontol 2000. 2006; $42: 47-79$

23. Peyyala R, Kirakodu SS, Ebersole JL, et al. Novel model for multispecies biofilms that uses rigid gas-permeable lenses. Appl Environ Microbiol. 2011;77:3413-21.

24. Lemon KP, Earl AM, Vlamakis HC, et al. Biofilm development with an emphasis on Bacillus subtilis. Curr Top Microbiol Immunol. 2008;322:1-16.

25. Jenkinson HF, Lamont RJ. Oral microbial communities in sickness and in health. Trends Microbiol. 2005;13:589-95.

26. Aas JA, Paster BJ, Stokes LN, et al. Defining the normal bacterial flora of the oral cavity. J Clin Microbiol. 2005;43:5721-32.

27. Lucas VS, Beighton D, Roberts GJ. Composition of the oral streptococcal flora in healthy children. J Dent. 2000;28:45-50.

28. Duran-Pinedo AE, Baker VD, Frias-Lopez J. The periodontal pathogen Porphyromonas gingivalis induces expression of transposases and cell death of Streptococcus mitis in a biofilm model. Infect Immun. 2014;82:3374-82.

29. Mashima I, Nakazawa F. The influence of oral Veillonella species on biofilms formed by Streptococcus species. Anaerobe. 2014;28: 54-61.

30. Teles RP, Haffajee AD, Socransky SS. Microbiological goals of periodontal therapy. Periodontol 2000. 2006;42:180-218.

31. Socransky SS, Haffajee AD. Periodontal microbial ecology. Periodontol 2000. 2005;38:135-87.

32. Haffajee AD, Socransky SS. Microbial etiological agents of destructive periodontal diseases. Periodontol 2000. 1994;5:78-111.

33. Matarazzo F, Figueiredo LC, Cruz SE, et al. Clinical and microbiological benefits of systemic metronidazole and amoxicillin in the treatment of smokers with chronic periodontitis: a randomized placebo-controlled study. J Clin Periodontol. 2008;35:885-96.

34. Socransky SS. Relationship of bacteria to the etiology of periodontal disease. J Dent Res. 1970;49:203-22.

35. Ritz HL. Microbial population shifts in developing human dental plaque. Arch Oral Biol. 1967;12:1561-8.

36. Theilade E, Wright WH, Jensen SB, et al. Experimental gingivitis in man. II. A longitudinal clinical and bacteriological investigation. J Periodontal Res. 1966;1:1-13.

37. Haffajee AD, Socransky SS, Patel MR, et al. Microbial complexes in supragingival plaque. Oral Microbiol Immunol. 2008;23:196205.

38.• Haffajee AD, Teles RP, Patel MR, et al. Factors affecting human supragingival biofilm composition. I. Plaque mass. J Periodontal Res. 2009;44:511-9.

39. Lee A, Ghaname CB, Braun TM, et al. Bacterial and salivary biomarkers predict the gingival inflammatory profile. J Periodontol. 2012;83:79-89.

40. Li J, Helmerhorst EJ, Leone CW, et al. Identification of early microbial colonizers in human dental biofilm. J Appl Microbiol. 2004;97:1311-8.

41. Sachdeo A, Haffajee AD, Socransky SS. Biofilms in the edentulous oral cavity. J Prosthodont. 2008;17:348-56.

42. Teles FR, Teles RP, Sachdeo A, et al. Comparison of microbial changes in early redeveloping biofilms on natural teeth and dentures. J Periodontol. 2012;83:1139-48. 
43. Uzel NG, Teles FR, Teles RP, et al. Microbial shifts during dental biofilm re-development in the absence of oral hygiene in periodontal health and disease. J Clin Periodontol. 2011;38:612-20.

44. Hellstrom MK, Ramberg P, Krok L, et al. The effect of supragingival plaque control on the subgingival microflora in human periodontitis. J Clin Periodontol. 1996;23:934-40.

45. Westfelt E, Rylander H, Dahlen G, et al. The effect of supragingival plaque control on the progression of advanced periodontal disease. J Clin Periodontol. 1998;25:536-41.

46. Ribeiro EP, Bittencourt S, Nociti-Junior FH, et al. The effect of one session of supragingival plaque control on clinical and biochemical parameters of chronic periodontitis. J Appl Oral Sci. 2005;13:275-9.

47. Kho P, Smales FC, Hardie JM. The effect of supragingival plaque control on the subgingival microflora. J Clin Periodontol. 1985;12: 676-86.

48. Beltrami M, Bickel M, Baehni PC. The effect of supragingival plaque control on the composition of the subgingival microflora in human periodontitis. J Clin Periodontol. 1987;14:161-4.

49. Katsanoulas T, Renee I, Attstrom R. The effect of supragingival plaque control on the composition of the subgingival flora in periodontal pockets. J Clin Periodontol. 1992;19:760-5.

50. Socransky SS, Haffajee AD, Cugini MA, et al. Microbial complexes in subgingival plaque. J Clin Periodontol. 1998;25:134-44.

51. Teles R, Sakellari D, Teles F, et al. Relationships among gingival crevicular fluid biomarkers, clinical parameters of periodontal disease, and the subgingival microbiota. J Periodontol. 2010;81:89-98.

52. Haffajee AD, Teles RP, Patel MR, et al. Factors affecting human supragingival biofilm composition. I. Plaque mass. J Periodontal Res. 2009;44:511-9.

53. Quirynen M, Bollen CM. The influence of surface roughness and surface-free energy on supra- and subgingival plaque formation in man. A review of the literature. J Clin Periodontol. 1995;22:1-14.

54. Quirynen M, Marechal M, Busscher HJ, et al. The influence of surface free-energy on planimetric plaque growth in man. J Dent Res. 1989;68:796-9.

55. Souto R, Silva-Boghossian CM, Colombo AP. Prevalence of Pseudomonas aeruginosa and Acinetobacter spp. in subgingival biofilm and saliva of subjects with chronic periodontal infection. Braz J Microbiol. 2014;45:495-501.

56. Ximenez-Fyvie LA, Haffajee AD, Socransky SS. Microbial composition of supra- and subgingival plaque in subjects with adult periodontitis. J Clin Periodontol. 2000;27:722-32.

57. Ximenez-Fyvie LA, Haffajee AD, Socransky SS. Comparison of the microbiota of supra- and subgingival plaque in health and periodontitis. J Clin Periodontol. 2000;27:648-57.

58. Griffen AL, Beall CJ, Campbell JH, et al. Distinct and complex bacterial profiles in human periodontitis and health revealed by $16 \mathrm{~S}$ pyrosequencing. ISME J. 2012;6:1176-85.

59. Mysak J, Podzimek S, Sommerova P, et al. Porphyromonas gingivalis: major periodontopathic pathogen overview. J Immunol Res. 2014;2014:476068

60. Jentsch HF, Marz D, Kruger M. The effects of stress hormones on growth of selected periodontitis related bacteria. Anaerobe. 2013;24:49-54.

61. Thurnheer T, Belibasakis GN, Bostanci N. Colonisation of gingival epithelia by subgingival biofilms in vitro: role of "red complex" bacteria. Arch Oral Biol. 2014;59:977-86.

62. Abusleme L, Dupuy AK, Dutzan N, et al. The subgingival microbiome in health and periodontitis and its relationship with community biomass and inflammation. ISME J. 2013;7:1016-25.

63. Hojo K, Nagaoka S, Ohshima T, et al. Bacterial interactions in dental biofilm development. J Dent Res. 2009;88:982-90.

64. Egland PG, Palmer Jr RJ, Kolenbrander PE. Interspecies communication in Streptococcus gordonii-Veillonella atypica biofilms: signaling in flow conditions requires juxtaposition. Proc Natl Acad Sci U S A. 2004;101:16917-22.
65. Brown SA, Whiteley M. A novel exclusion mechanism for carbon resource partitioning in Aggregatibacter actinomycetemcomitans. J Bacteriol. 2007;189:6407-14.

66. Mayrand D, McBride BC. Exological relationships of bacteria involved in a simple, mixed anaerobic infection. Infect Immun. 1980;27:44-50.

67. Grenier D, Mayrand D. Studies of mixed anaerobic infections involving Bacteroides gingivalis. Can J Microbiol. 1983;29:612-8.

68. Grenier D. Nutritional interactions between two suspected periodontopathogens, Treponema denticola and Porphyromonas gingivalis. Infect Immun. 1992;60:5298-301.

69. Aruni AW, Roy F, Sandberg L, et al. Proteome variation among Filifactor alocis strains. Proteomics. 2012;12:3343-64.

70. Aruni AW, Zhang K, Dou Y, et al. Proteome analysis of coinfection of epithelial cells with Filifactor alocis and Porphyromonas gingivalis shows modulation of pathogen and host regulatory pathways. Infect Immun. 2014;82:3261-74.

71. Uematsu H, Sato N, Hossain MZ, et al. Degradation of arginine and other amino acids by butyrate-producing asaccharolytic anaerobic Gram-positive rods in periodontal pockets. Arch Oral Biol. 2003;48:423-9.

72. Eley BM, Cox SW. Cathepsin B/L-, elastase-, tryptase-, trypsinand dipeptidyl peptidase IV-like activities in gingival crevicular fluid: a comparison of levels before and after periodontal surgery in chronic periodontitis patients. J Periodontol. 1992;63:412-7.

73. Bramanti TE, Holt SC. Roles of porphyrins and host iron transport proteins in regulation of growth of Porphyromonas gingivalis W50. J Bacteriol. 1991;173:7330-9.

74. Kornman KS, Loesche WJ. Effects of estradiol and progesterone on Bacteroides melaninogenicus and Bacteroides gingivalis. Infect Immun. 1982;35:256-63.

75. Turnbaugh PJ, Ley RE, Hamady M, et al. The human microbiome project. Nature. 2007;449:804-10.

76. Chen $\mathrm{T}, \mathrm{Yu} \mathrm{WH}$, Izard $\mathrm{J}$ et al. The Human Oral Microbiome Database: a web accessible resource for investigating oral microbe taxonomic and genomic information. Database (Oxford). 2010;2010:baq013.

77. Griffen AL, Beall CJ, Firestone ND, et al. CORE: a phylogenetically-curated $16 \mathrm{~S}$ rDNA database of the core oral microbiome. PLoS One. 2011;6:e19051.

78. Dewhirst FE, Chen T, Izard J, et al. The human oral microbiome. J Bacteriol. 2010;192:5002-17.

79. Saito A, Inagaki S, Ishihara K. Differential ability of periodontopathic bacteria to modulate invasion of human gingival epithelial cells by Porphyromonas gingivalis. Microb Pathog. 2009;47:329-33.

80. Soro V, Dutton LC, Sprague SV, et al. Axenic Culture of a Candidate Division TM7 Bacterium from the Human Oral Cavity and Biofilm Interactions with Other Oral Bacteria. Appl Environ Microbiol. 2014;80:6480-9.

81. Aruni AW, Roy F, Fletcher HM. Filifactor alocis has virulence attributes that can enhance its persistence under oxidative stress conditions and mediate invasion of epithelial cells by Porphyromonas gingivalis. Infect Immun. 2011;79:3872-86.

82. Fine DH, Markowitz K, Fairlie K et al. A consortium of Aggregatibacter actinomycetemcomitans (Aa), Streptococcus parasanguinis and Filifactor alocis are present in sites prior to bone loss in a longitudinal study of localized aggressive periodontitis. J Clin Microbiol. 2013.

83. Slots J. Human viruses in periodontitis. Periodontol 2000. 2010;53: 89-110.

84. Thoulouze MI, Alcover A. Can viruses form biofilms? Trends Microbiol. 2011;19:257-62.

85. Pais-Correia AM, Sachse M, Guadagnini S, et al. Biofilm-like extracellular viral assemblies mediate HTLV-1 cell-to-cell transmission at virological synapses. Nat Med. 2010;16:83-9. 
86. He J, Li Y, Cao Y et al. The oral microbiome diversity and its relation to human diseases. Folia Microbiol (Praha). 2014.

87. Miller CS. Viruses: are they really culprits for periodontal disease? A critical review? J Investig Clin Dent. 2014;5:243.

88. Maddi A, Scannapieco FA. Oral biofilms, oral and periodontal infections, and systemic disease. Am J Dent. 2013;26:249-54.

89. Cappuyns I, Gugerli P, Mombelli A. Viruses in periodontal disease a review. Oral Dis. 2005;11:219-29.

90. Stein RA. Epigenetics-the link between infectious diseases and cancer. JAMA. 2011;305:1484-5.
91. Minarovits J. Microbe-induced epigenetic alterations in host cells: the coming era of patho-epigenetics of microbial infections. A review. Acta Microbiol Immunol Hung. 2009;56:1-19.

92. Paschos K, Allday MJ. Epigenetic reprogramming of host genes in viral and microbial pathogenesis. Trends Microbiol. 2010;18:439-47.

93. Niller HH, Banati F, Nagy K, et al. Update on microbe- incudced epigenetic changes: bacterial effectors and viral oncogenes. Futur Virol. 2013;8:1111-26.

94. McCullers JA. The co-pathogenesis of influenza viruses with bacteria in the lung. Nat Rev Microbiol. 2014;12:252-62. 